\title{
THE RANDOM CONNECTION MODEL ON THE TORUS
}

\author{
LUC DEVROYE AND NICOLAS FRAIMAN
}

\begin{abstract}
We study the diameter of a family of random graphs on the torus that can be used to model wireless networks. In the random connection model two points $x$ and $y$ are connected with probability $g(y-x)$ where $g$ is a given function. We prove that the diameter of the graph is bounded by a constant, that only depends on $\|g\|_{1}$, with high probability as the number of vertices in the graph tends to infinity.
\end{abstract}

\section{INTRODUCTION}

The idea of modeling networks using random graphs appeared for the first time in Gilbert (1961) where he considered a network formed by connecting points of a Poisson point process that are sufficiently close to each other. His paper marks the starting point for continuum percolation. He proved the existence of a critical distance above which an infinite component occurs and below which any connected component is bounded.

The random connection model was introduced in the context of continuum percolation by Penrose (1991). Let $g: \mathbb{R}^{2} \rightarrow[0,1]$ be such that $g(-x)=g(x)$. The function $g$ is called the connection function. Let $\mathcal{P}$ be a homogeneous Poisson point process in the plane of intensity $\lambda>0$ and connect every pair of points $x, y \in \mathcal{P}$ independently with probability $g(y-x)$. Typically it is also assumed that $g$ only depends on the distance between $x$ and $y$, that is $g(y-x)=\tilde{g}(\|y-x\|)$ where $\tilde{g}: \mathbb{R}^{+} \rightarrow[0,1]$ and $\|\cdot\|$ denotes the Euclidean norm. Moreover, in general $\tilde{g}$ is taken to be nonincreasing. Gilbert's graph is the special case of the random connection model with $\tilde{g}=\mathbf{1}_{[0,1]}$.

The main result that motivates the study of the model is that provided $\lambda$ exceeds a critical value $\lambda_{g}$ depending on $g$, namely the percolation threshold, the random connection model has an infinite component almost surely. For a survey on continuum percolation, see the book by Meester and Roy (1996).

The random connection model is a reasonable approximation of a wireless communication network where nodes cooperate in routing each other's packets from source to destination in a multi-hop way (see Franceschetti and Meester, 2008). Points represent transmission stations and links represent communication channels. A common assumption is that links become less reliable as the distance between nodes increases. Another natural application is to the study of spread of a contagious

Date: March 11, 2013

2010 Mathematics Subject Classification. 60C05, 05C80.

Key words and phrases. random graphs, random connection model, diameter.

The research of both authors was sponsored by NSERC Grant A3456. 
disease. Here the points represent individuals susceptible to the disease, the function $g$ represents the probability of contagion, and the disease spreads from an infected individual to other individuals along the links of the network.

When we say that a property of the graph holds with high probability, we mean that the probability that the property does not hold is bounded by a function of $n$ that goes to zero as $n \rightarrow \infty$. Equivalently, we say that a sequence of random events $E_{n}$ occurs with high probability if $\lim _{n \rightarrow \infty} \mathbf{P}\left(E_{n}\right)=1$.

In this short note we prove that when we restrict the model to the torus, which we denote by $\mathcal{T}^{d}$, with high probability the graph is not only connected, but also the diameter of the graph is bounded by a constant, only depending on $\|g\|_{1}$, as the number of vertices in the graph tends to infinity. It is interesting to note the lack of conditions for $g$. The proof works for any measurable function $g: \mathcal{T}^{d} \rightarrow[0,1]$ that is not identically zero. In our case, we build the graph on $n$ uniformly distributed points rather than a Poisson point process but the results are equivalent for the Poisson case when the intensity $\lambda \rightarrow \infty$.

Our argument is based on the existence of disjoint paths starting at a given origin vertex. If the paths are long enough, the locations of the endpoints of these paths are close to uniform on $\mathcal{T}^{d}$ and we can therefore approximate the probability that they connect to a destination vertex. The paper is organized as follows: In Section 2 we introduce the model. In Section 3 we prove the convergence to the uniform distribution via Fourier analysis. The proof of the main result is done in Section 4. Finally, in Section 5 we note possible generalizations.

\section{THE MODEL ON THE TORUS}

Denote the circle by $\mathcal{S}^{1}=[0,1)$ and the $d$-dimensional torus by $\mathcal{T}^{d}=\left(\mathcal{S}^{1}\right)^{d}$. $\mathcal{T}^{d}$ is a compact Abelian Lie group and given two elements $x, y \in \mathcal{T}^{d}$ we write $x+y$ for the group operation. Let $g: \mathcal{T}^{d} \rightarrow[0,1]$ be a measurable function satisfying $g(-x)=g(x)$ and $g \neq 0$.

Consider a random graph on $\mathcal{T}^{d}$ defined as follows. Let $X_{1}, \ldots, X_{n}$ be independent random points of $\mathcal{T}^{d}$ with a common uniform distribution. These points are associated with the vertices $V_{n}=\{1, \ldots, n\}$ of a graph $G(n, g)=\left(V_{n}, E_{n}\right)$. With every pair $i, j \in V_{n}, i \neq j$, associate an independent uniform $[0,1]$ random variable $U_{i j}$. A pair $(i, j)$ defines an edge of $E_{n}$ if

$$
U_{i j} \leq g\left(X_{j}-X_{i}\right) .
$$

Let $Y_{i j}=\mathbf{1}_{(i, j) \in E_{n}}$ and write $D_{i}=\sum_{j \neq i} Y_{i j}$ for the degree of vertex $i$. Note that, by linearity, the expected degree of any vertex is given by $\mathbf{E} D_{i}=\|g\|_{1}(n-1)$ where $\|g\|_{1}=\int_{\mathcal{T}^{d}} g(x) \mathrm{d} x$ because using that the uniform measure is the Haar measure of $\mathcal{T}^{d}$, i.e., it is translation invariant, we have

$$
\mathbf{E} Y_{i j}=\mathbf{E}\left[g\left(X_{i}-X_{j}\right)\right]=\int_{\mathcal{T}^{d}} \int_{\mathcal{T}^{d}} g(y-x) \mathrm{d} y \mathrm{~d} x=\int_{\mathcal{T}^{d}}\|g\|_{1} \mathrm{~d} x=\|g\|_{1} .
$$

We prove that with high probability we can find a uniformly bounded length path between any pair of vertices, in which the length depends only on $\|g\|_{1}$. Define 
the diameter of the graph as

$$
\operatorname{diam} G(n, g)= \begin{cases}\sup _{p, q \in V_{n}} \operatorname{dist}_{G}(p, q) & \text { if the graph is connected } \\ \infty & \text { otherwise. }\end{cases}
$$

Therefore, with high probability the diameter of $G(n, g)$ is bounded by a constant. The main result of this note is the following

Theorem 1. Let $g: \mathcal{T}^{d} \rightarrow[0,1]$ be a measurable function with $\|g\|_{1}>0$, and define $m=2+\left\lceil\left(1 /\|g\|_{1}^{2}\right) \log \left(1 / 2\|g\|_{1}^{2}\right)\right\rceil$ and $\gamma=\|g\|_{1}^{m+1} / 4 m$. Then, for all $n \geq m+2$ we have

$$
\mathbf{P}(\operatorname{diam} G(n, g) \geq m+1) \leq n^{2} e^{-\gamma n} .
$$

The following corollary follows immediately.

Corollary 2. If $g_{n}$ depends on $n$, and $m_{n}$ and $\gamma_{n}$ are defined as above, the conditions $n \geq m_{n}+2$ and $\gamma_{n} n-2 \log n \rightarrow \infty$ imply $G\left(n, g_{n}\right)$ is connected with high probability.

\section{RATE of CONVERGENCE OF CONVOLUTIONS}

In this section we show a exponential bound for the rate of convergence to the uniform density of the convolution of bounded densities on $\mathcal{T}^{d}$. The technique we use is based on harmonic analysis. As a general reference for the basic results in this whole section, see Grafakos (2008). We first prove an upper bound on the Fourier coefficients of a bounded density. We follow the standard notation and write for $f \in L^{2}\left(\mathcal{T}^{d}\right)$

$$
f(x)=\sum_{p \in \mathbb{Z}^{d}} c_{p}(f) e^{2 \pi i\langle p, x\rangle} .
$$

where $\langle\cdot, \cdot\rangle$ is the inner product obtained by identifying $\mathcal{T}^{d}$ with $[0,1)^{d}$ in $\mathbb{R}^{d}$ and

$$
c_{p}(f)=\int_{\mathcal{T}^{d}} f(x) e^{-2 \pi i\langle p, x\rangle} \mathrm{d} x .
$$

Note that if $f \geq 0$, then $c_{0}(f)=\|f\|_{1}$. Denote the family of densities bounded by $B \geq 1$ by

$$
\mathcal{F}_{B}=\left\{f: \mathcal{T}^{d} \rightarrow[0, B],\|f\|_{1}=1\right\} .
$$

It is clear that $\mathcal{F}_{B} \subset L^{\infty}\left(\mathcal{T}^{d}\right) \subset L^{2}\left(\mathcal{T}^{d}\right)$.

Lemma 3. For all $p \in \mathbb{Z}^{d} \backslash\{0\}$ and $B \geq 1$,

$$
\sup _{f \in \mathcal{F}_{B}}\left|c_{p}(f)\right|=\frac{B}{\pi} \sin \left(\frac{\pi}{B}\right) \text {. }
$$

Proof. Let $p \in \mathbb{Z}^{d} \backslash\{0\}$ be fixed. Given $f \in \mathcal{F}_{B}$ we compare the coefficient $c_{p}(f)$ with the corresponding one of a function of the form $h(x)=B \mathbf{1}_{\mathcal{U}}$ for a set $\mathcal{U}$ depending on $f$. There exists $\theta \in[0,1)$ such that

$$
\left|c_{p}(f)\right|=e^{2 \pi i \theta} \int_{\mathcal{T}^{d}} f(x) e^{-2 \pi i\langle p, x\rangle} \mathrm{d} x=\int_{\mathcal{T}^{d}} f(x) \varphi(x) \mathrm{d} x,
$$

where we write $\varphi(x)=\cos 2 \pi(\langle p, x\rangle-\theta)$. The second equality above follows from considering the real part and the fact that $\left|c_{p}(f)\right| \geq 0$. 
Note that the level sets of $\varphi(x)$ are of the form $\left\{x \in \mathcal{T}^{d}:\langle p, x\rangle-c \in \mathbb{Z}\right\}$ for some constant $c \in[0,1)$. In particular the maximum value is attained in

$$
\mathcal{L}=\left\{x \in \mathcal{T}^{d}:\langle p, x\rangle-\theta \in \mathbb{Z}\right\} .
$$

If $p=\left(p_{1}, \ldots, p_{d}\right)$, we let $p=\ell q$ where $\ell=\operatorname{gcd}\left(p_{1}, \ldots, p_{d}\right)$ and $q=\left(q_{1}, \ldots, q_{d}\right)$ with $q_{1}, \ldots, q_{d}$ coprime. Then, $\mathcal{L}=\cup_{k=1}^{\ell} \mathcal{H}_{k}$ where

$$
\mathcal{H}_{k}=\left\{x \in \mathcal{T}^{d}:\langle q, x\rangle-\theta / \ell-k / \ell \in \mathbb{Z}\right\}
$$

and each connected component $\mathcal{H}_{k}$ is a $(d-1)$-dimensional submanifold of measure $\mu\left(\mathcal{H}_{k}\right)=\|q\|$. Hence the total measure is $\mu(\mathcal{L})=\sum_{k=1}^{\ell} \mu\left(\mathcal{H}_{k}\right)=\|p\|$. Define

$$
\mathcal{U}=\left\{x \in \mathcal{T}^{d}: \operatorname{dist}(x, \mathcal{L}) \leq 1 / 2 B\|p\|\right\} .
$$

We prove that the supremum is attained when $h(x)=B \mathbf{1}_{\mathcal{U}}$.

Note that if $x \in \mathcal{U}$ and $y \in \mathcal{T}^{d} \backslash \mathcal{U}$ then $\varphi(x) \geq \varphi(y)$. Since both $h, f \in \mathcal{F}_{B}$ then $\int_{\mathcal{U}} B \mathrm{~d} x=1=\int_{\mathcal{T}^{d}} f(x) \mathrm{d} x$ and we have $\int_{\mathcal{U}} B-f(x) \mathrm{d} x=\int_{\mathcal{T}^{d} \backslash \mathcal{U}} f(x) \mathrm{d} x$. Therefore,

$$
\begin{aligned}
\left|c_{p}(h)\right|-\left|c_{p}(f)\right| & =\int_{\mathcal{T}^{d}}\left(B \mathbf{1}_{\mathcal{U}}-f(x)\right) \varphi(x) \mathrm{d} x \\
& =\int_{\mathcal{U}}(B-f(x)) \varphi(x) \mathrm{d} x-\int_{\mathcal{T}^{d} \backslash \mathcal{U}} f(x) \varphi(x) \mathrm{d} x \\
& \geq\left(\int_{\mathcal{U}} B-f(x) \mathrm{d} x\right) \inf _{x \in \mathcal{U}} \varphi(x)-\left(\int_{\mathcal{T}^{d} \backslash \mathcal{U}} f(x) \mathrm{d} x\right) \sup _{y \in \mathcal{T}^{d} \backslash \mathcal{U}} \varphi(y) \geq 0 .
\end{aligned}
$$

Therefore, the coefficient is maximized for $h$. We just compute the supremum now. Note that $\mathcal{U}=\cup_{k=1}^{\ell} \mathcal{V}_{k}$ where $\mathcal{V}_{k}=\left\{x \in \mathcal{T}^{d}: \operatorname{dist}\left(x, \mathcal{H}_{k}\right) \leq 1 / 2 B\|p\|\right\}$. Then,

$$
\begin{aligned}
\left|c_{p}(h)\right| & =\int_{\mathcal{U}} B \varphi(x) \mathrm{d} x \\
& =B \sum_{k=1}^{\ell} \mu\left(\mathcal{H}_{k}\right) \int_{-1 / 2 B\|p\|}^{1 / 2 B\|p\|} \cos (2 \pi\|p\| t) \mathrm{d} t \\
& =B \sum_{k=1}^{\ell}\|q\| \frac{1}{\pi\|p\|} \sin \left(\frac{\pi}{B}\right) \\
& =\frac{B}{\pi} \sin \left(\frac{\pi}{B}\right)
\end{aligned}
$$

which concludes the proof.

Given two functions $f, h \in L^{2}\left(\mathcal{T}^{d}\right)$, their convolution is given by

$$
f * h(x)=\int_{\mathcal{T}^{d}} f(y) h(x-y) \mathrm{d} y .
$$

Recall the relation between convolutions and Fourier coefficients given by

$$
c_{p}(f * h)=c_{p}(f) c_{p}(h) .
$$

Let $f \in L^{2}\left(\mathcal{T}^{d}\right)$ and write $f^{(k)}=f * f * \cdots * f$ for the $k$-fold convolution of $f$. Note that $c_{p}\left(f^{(k)}\right)=c_{p}(f)^{k}$. The main result in this section is the following. 
Lemma 4. Let $f \in \mathcal{F}_{B}$. Then

$$
\sup _{x \in \mathcal{T}^{d}}\left|1-f^{(k)}(x)\right| \leq B\left(\frac{B}{\pi} \sin \left(\frac{\pi}{B}\right)\right)^{k-2} .
$$

Proof. For all $f \in \mathcal{F}_{B}$, the coefficient $c_{0}(f)=\|f\|_{1}=1$. Therefore,

$$
\left|1-f^{(k)}(x)\right|=\left|\sum_{p \in \mathbb{Z}^{d} \backslash\{0\}} c_{p}(f)^{k} e^{2 \pi i\langle p, x\rangle}\right| \leq \sum_{p \in \mathbb{Z}^{d} \backslash\{0\}}\left|c_{p}(f)\right|^{k} .
$$

We can split the terms and apply Parseval's identity together with Lemma 3 to obtain

$$
\begin{aligned}
\left|1-f^{(k)}(x)\right| & \leq \sum_{p \in \mathbb{Z}^{d} \backslash\{0\}}\left|c_{p}(f)\right|^{2}\left|c_{p}(f)\right|^{k-2} . \\
& \leq \sum_{p \in \mathbb{Z}^{d} \backslash\{0\}}\left|c_{p}(f)\right|^{2}\left(\frac{B}{\pi} \sin \left(\frac{\pi}{B}\right)\right)^{k-2} . \\
& \leq\|f\|_{2}\left(\frac{B}{\pi} \sin \left(\frac{\pi}{B}\right)\right)^{k-2} .
\end{aligned}
$$

Since $f \in \mathcal{F}_{B}$, we have $\|f\|_{2} \leq\|f\|_{\infty} \leq B$, and that concludes the proof.

\section{A BOUND FOR THE DIAMETER}

In this section we prove the main result of this note. We begin by proving a lemma about the distribution of the position on $\mathcal{T}^{d}$ of the end vertex of a path in $G(n, g)$ started in any given vertex. Introduce $g_{k}=\left(g /\|g\|_{1}\right)^{(k)}$, the $k$-fold convolution of $g /\|g\|_{1}$. Given a fixed sequence of vertices $j_{0}, \ldots, j_{m}$, let $E\left(j_{0}, \ldots, j_{m}\right)=\cap_{k=1}^{m}\left\{\left(j_{k-1}, j_{k}\right) \in E_{n}\right\}$ denote the event that $j_{0}, \ldots, j_{m}$ is a path in $G(n, g)$.

Lemma 5. The location of the end vertex of a path of length $m$ in $G(n, g)$ has a distribution with density given by $g_{m}$. Formally, if $E\left(j_{0}, \ldots, j_{m}\right)$ occurs and $W=X_{j_{m}}-X_{j_{0}}$, the density

$$
f_{W}\left(x \mid E\left(j_{0}, \ldots, j_{m}\right)\right)=g_{m}(x), \quad x \in \mathcal{T}^{d} .
$$

Proof. Let $Z_{k}=X_{j_{k}}-X_{j_{k-1}}$. Note that $W=\sum_{k=1}^{m} Z_{k}$. Also, each $Z_{k}$ is uniformly distributed on $\mathcal{T}^{d}$ because it is the difference of two uniformly distributed points. By conditioning with respect to $X_{1}, \ldots, X_{n}$, we have

$$
\mathbf{P}\left(E\left(j_{0}, \ldots, j_{m}\right)\right)=\mathbf{P}\left(\bigcap_{k=1}^{m}\left(j_{k-1}, j_{k}\right) \in E_{n}\right)=\mathbf{E}\left[\prod_{k=1}^{m} g\left(Z_{k}\right)\right]=\|g\|_{1}^{m} .
$$

Define the vector $Z=\left(Z_{1}, \ldots, Z_{m}\right)$ and write $u=\left(u_{1}, \ldots, u_{m}\right) \in\left(\mathcal{T}^{d}\right)^{m}$ for a vector of $m$ points in the torus. The conditional probability of the path given $Z=u$ is

$$
\mathbf{P}\left(E\left(j_{0}, \ldots, j_{m}\right) \mid Z=u\right)=\mathbf{E}\left[\prod_{k=1}^{m} g\left(Z_{k}\right) \mid Z=u\right]=\prod_{k=1}^{m} g\left(u_{k}\right) .
$$


The distribution of $Z$ is uniform on $\left(\mathcal{T}^{d}\right)^{m}$ because each $Z_{k}$ it uniformly distributed on $\mathcal{T}^{d}$. Thus, we have $f_{Z}(u)=1$ for all $u \in\left(\mathcal{T}^{d}\right)^{m}$. Then, by Bayes' theorem, the conditional density of $Z$ given that $j_{0}, \ldots, j_{m}$ is a path in $G(n, g)$ is

$$
f_{Z}\left(u \mid E\left(j_{0}, \ldots, j_{m}\right)\right)=\frac{\mathbf{P}\left(E\left(j_{0}, \ldots, j_{m}\right) \mid Z=u\right) f_{Z}(u)}{\mathbf{P}\left(E\left(j_{0}, \ldots, j_{m}\right)\right)}=\frac{\prod_{k=1}^{m} g\left(u_{k}\right) \cdot 1}{\|g\|_{1}^{m}} .
$$

Note that by similar arguments $h_{k}(x)=g(x) /\|g\|_{1}$ is the conditional density of $Z_{k}$. Hence, the variables $Z_{1}, \ldots, Z_{m}$ are conditionally independent given that $E\left(j_{0}, \ldots, j_{m}\right)$ occurs. Therefore, the conditional density of the sum $W=\sum_{k=1}^{m} Z_{k}$ is given by

$$
f_{W}\left(x \mid E\left(j_{0}, \ldots, j_{m}\right)\right)=\left(h_{1} * \cdots * h_{m}\right)(x)=g_{m}(x) .
$$

We want to bound the probability that a fixed pair of vertices $i, j$ is not connected by a path of bounded length. The idea behind the proof is to construct a large number of disjoint long paths starting in $i$ and then use the fact that the end points of these paths are independent and relatively close to being uniformly distributed on $\mathcal{T}^{d}$ to show that the probability that none of them connects with $j$ decays exponentially.

Proposition 6. Let $m=2+\left[\left(1 /\|g\|_{1}^{2}\right) \log \left(1 / 2\|g\|_{1}^{2}\right)\right\rceil$ and $\gamma=\|g\|_{1}^{m+1} / 4 m$. Then for all $i, j \in V_{n}$, and all $n \geq m+2$ we have

$$
\mathbf{P}\left(\operatorname{dist}_{G}(i, j)>m+1\right) \leq e^{-\gamma n} .
$$

Proof. We look for paths of length $m+1$ between any pair of vertices in the graph. To simplify the notation we look at $\operatorname{dist}_{G}(n-1, n)$. Let $\ell=\lfloor(n-2) / m\rfloor$ and for $s=0, \ldots, \ell-1$, consider the events

$$
\begin{aligned}
A_{s, 0} & =\left\{(n-1, s m+1) \in E_{n}\right\}, \\
A_{s, k} & =\left\{(s m+k, s m+k+1) \in E_{n}\right\}, \quad \text { for } k=1, \ldots, m-1 \\
A_{s, m} & =\left\{(s m+m, n) \in E_{n}\right\} .
\end{aligned}
$$

Let $F_{s}=\cap_{k=0}^{m-1} A_{s, k}$ be the event that $n-1, s m+1, \ldots, s m+m$ is a path in $G(n, g)$ and $C_{s}=F_{s} \cap A_{s, m}$ the event that this path connects $n-1$ to $n$. Note that

$$
\begin{aligned}
\mathbf{P}\left(\bigcap_{s=0}^{\ell-1} C_{s}^{c}\right) & =\mathbf{E}\left[\mathbf{P}\left(\bigcap_{s=0}^{\ell-1} C_{s}^{c} \mid X_{n-1}, X_{n}\right)\right] \\
& =\mathbf{E}\left[\prod_{s=0}^{\ell-1} \mathbf{P}\left(C_{s}^{c} \mid X_{n-1}, X_{n}\right)\right],
\end{aligned}
$$

since for different values of $s$ the events $C_{s}$ involve disjoint subsets of vertices (except the two endpoints $n-1$ and $n$ ). For any $s$ the conditional probability above can be split as follows:

$$
\begin{aligned}
\mathbf{P}\left(C_{s}^{c}\right. & \left.\mid X_{n-1}, X_{n}\right) \\
& =\mathbf{P}\left(F_{s}^{c} \mid X_{n-1}, X_{n}\right)+\mathbf{P}\left(A_{s, m}^{c} \mid F_{s}, X_{n-1}, X_{n}\right) \mathbf{P}\left(F_{s} \mid X_{n-1}, X_{n}\right) .
\end{aligned}
$$

Note $F_{s}=E(n-1, s m+1, \ldots, s m+m)$ is independent of $X_{n}$ and the value of $X_{n-1}$ does not change the probability of $F_{s}$ by translation invariance. Therefore, 
the conditional probability of having the given path in $G(n, g)$ is equal to

$$
\mathbf{P}\left(F_{s} \mid X_{n-1}, X_{n}\right)=\|g\|_{1}^{m} .
$$

To compute the probability of making the last connection, let $Z_{s}=X_{s m+m}-$ $X_{n-1}$. Note that, by Lemma 5, conditional on $F_{s}$, the difference $Z_{s}$ is distributed with density given by $g_{m}$. Since $g: \mathcal{T}^{d} \rightarrow[0,1]$ we have that

$$
\frac{g(x)}{\|g\|_{1}} \leq \frac{1}{\|g\|_{1}} \quad \text { so } \quad \frac{g}{\|g\|_{1}} \in \mathcal{F}_{\|g\|_{1}^{-1}} .
$$

Then, by Lemma 4 we have

$$
\sup _{x \in \mathcal{T}^{d}}\left|1-g_{m}(x)\right| \leq \frac{1}{\|g\|_{1}}\left(\frac{\sin \pi\|g\|_{1}}{\pi\|g\|_{1}}\right)^{m-2} \leq \frac{1}{\|g\|_{1}} e^{-\|g\|_{1}^{2}(m-2)} \leq \frac{\|g\|_{1}}{2} .
$$

The second inequality follows from the fact that $(\sin \pi t) / \pi t \leq e^{-t^{2}}$ for $t \in[0,1]$ and the last inequality holds by the definition of $m$. Therefore,

$$
\begin{aligned}
\mathbf{P}\left(A_{s, m}^{c} \mid F_{s}, X_{n-1}, X_{n}\right) & =\mathbf{E}\left[1-g\left(X_{n}-Z_{s}-X_{n-1}\right) \mid F_{s}, X_{n-1}, X_{n}\right] \\
& =\mathbf{E}\left[1-g\left(Z_{s}\right) \mid F_{s}\right] \\
& =\int_{\mathcal{T}^{d}}(1-g(x)) g_{m}(x) \mathrm{d} x . \\
& =1-\|g\|_{1}+\int_{\mathcal{T}^{d}}(1-g(x))\left(g_{m}(x)-1\right) \mathrm{d} x . \\
& \leq 1-\frac{\|g\|_{1}}{2} .
\end{aligned}
$$

Combining the bound above with the identity from equation (3) and substituting in equation (2) we obtain

$$
\mathbf{P}\left(C_{s}^{c} \mid X_{n-1}, X_{n}\right) \leq\left(1-\|g\|_{1}^{m}\right)+\left(1-\frac{\|g\|_{1}}{2}\right)\|g\|_{1}^{m}=1-\frac{\|g\|_{1}^{m+1}}{2} .
$$

Applying this bound in equation (1) we have

$$
\mathbf{P}\left(\operatorname{dist}_{G}(n-1, n)>m+1\right) \leq \mathbf{P}\left(\bigcap_{s=0}^{\ell-1} C_{s}^{c}\right) \leq\left(1-\frac{\|g\|_{1}^{m+1}}{2}\right)^{\ell} \leq e^{-\gamma n},
$$

where $\gamma=\|g\|_{1}^{m+1} / 4 m$ as in the statement of the lemma.

The main result is that any pair of vertices $i, j$ is connected by a path of bounded length with high probability. Below we state the theorem again. The proof follows immediately from Proposition 6 .

Theorem 1. Let $g: \mathcal{T}^{d} \rightarrow[0,1]$ be a measurable function with $\|g\|_{1}>0$, and define $m=2+\left\lceil\left(1 /\|g\|_{1}^{2}\right) \log \left(1 / 2\|g\|_{1}^{2}\right)\right\rceil$ and $\gamma=\|g\|_{1}^{m+1} / 4 m$. Then, for all $n \geq m+2$ we have

$$
\mathbf{P}(\operatorname{diam} G(n, g) \geq m+1) \leq n^{2} e^{-\gamma n} .
$$

Proof. By Proposition 6, and a union bound we have

$$
\mathbf{P}(\operatorname{diam} G(n, g)>m+1) \leq \sum_{i, j=1}^{n} \mathbf{P}\left(\operatorname{dist}_{G}(i, j)>m+1\right) \leq n^{2} e^{-\gamma n} .
$$




\section{Remarks And Generalizations}

The bounds in our results depend only upon $n$ and $\|g\|_{1}$ and should thus prove useful in many applications. For example, for functions $g$ that depend upon the norm of the argument, all norms can be dealt with at once in a homogeneous manner. If $g(x)=\prod_{i=1}^{d} h\left(x_{i}\right)$ is a product, with $h: \mathcal{S}^{1} \rightarrow[0,1]$ fixed, then $\|g\|_{1}=\left(\int h(x) \mathrm{d} x\right)^{d}$, so that the one could in principle let $d$ tend to infinity with $n$ without sacrificing connectivity. For example, connectivity occurs with high probability if $h$ is fixed and

$$
d<\frac{1-\varepsilon}{2} \cdot \frac{\log \log n}{-\log \left(\int h(x) \mathrm{d} x\right)}
$$

Note that our proof also works in the directed case, where we do not need $g(-x)=g(x)$. In this case it implies that the graph is strongly connected. Moreover, Since the bounds in Proposition 6 are explicit functions of $\|g\|_{1}$ we can use the same argument to show that a more general model, in which we have $g_{n}$ changing with $n$ still has bounded diameter as long as $\left\|g_{n}\right\|_{1}$ does not goes to zero too quickly. The bound is strong enough to obtain a connected graph with high probability for sequences $g_{n}$ such that $\left\|g_{n}\right\|_{1}>(\log n)^{-\beta}$ for some $0<\beta<1 / 2$.

\section{REFERENCES}

P. Flajolet and R. Sedgewick. Analytic Combinatorics. Cambridge University Press, 2009.

P. Flajolet, K. P. Hatzis, S. E. Nikoletseas, and P. G. Spirakis. On the robustness of interconnections in random graphs: a symbolic approach. Theoretical Computer Science, 287(2):515-534, 2002.

P. Flajolet, B. Salvy, and G. Schaeffer. Airy phenomena and analytic combinatorics of connected graphs. Electronic Journal of Combinatorics, 11(1):R34, 2004.

M. Franceschetti and R. Meester. Random Networks for Communication: From Statistical Physics to Information Systems. Number 24 in Cambridge Series in Statistical and Probabilistic Mathematics. Cambridge University Press, 2008.

E. Gilbert. Random plane networks. Journal of the Society for Industrial and Applied Mathematics, 9(4):533-543, 1961.

L. Grafakos. Classical Fourier Analysis. Graduate Texts in Mathematics. Springer, 2008.

J. C. Lagarias, A. M. Odlyzko, and D. B. Zagier. On the capacity of disjointly shared networks. Computer Networks and ISDN Systems, 10(5):275-285, 1985.

G. Louchard. Random walks, gaussian processes and list structures. Theoretical Computer Science, 53(1):99-124, 1987.

R. Meester and R. Roy. Continuum Percolation. Number 119 in Cambridge Tracts in Mathematics. Cambridge University Press, 1996.

M. Penrose. On a continuum percolation model. Advances in Applied Probability, 23(3):536-556, 1991. 
School of Computer Science, McGill University, Montreal, Canada H3A $2 \mathrm{~K} 6$.

E-mail address: luc@cs.mcgill.ca

Department of Mathematics and Statistics, McGill University, Montreal, Canada H3A $2 \mathrm{~K} 6$.

E-mail address: fraiman@math.mcgill.ca 